Supporting Online Information for

\title{
Swollen Structures of Brodie Graphite Oxide as Solid Solvates.
}

Anastasiya T. Rebrikova ${ }^{1}$, Alexey Klechikov, ${ }^{2,3}$ Artem Iakunkov, ${ }^{2}$ Jinhua Sun, ${ }^{2,4}$ Alexandr V. Talyzin $^{2}$, Natalya V. Avramenko ${ }^{1}$, Mikhail Korobov, ${ }^{1, *}$

${ }^{1}$ Department of Chemistry, Moscow State University, Leninskie Gory 1-3, Moscow 119991 , Russia

${ }^{2}$ Umeå University, Department of Physics, S-90187 Umeå, Sweden

${ }^{3}$ Uppsala University, Department of Physics and Astronomy, Materials Physics, Ångströmlaboratoriet, Lägerhyddsvägen 1, Box 516

75120 Uppsalla, Sweden

${ }^{4}$ Department of Industrial and Materials Science, Chalmers Tekniska Högskola, 41296 Göteborg, Sweden

*To whom correspondence should be addressed. E-mail: mkorobov49@gmail.com

\section{S1. Experimental}

S1.1 XPS spectra.

Five batches of BGO with slightly different degree of oxidation were used in this study, two BGO materials with 1 step oxidation and B-GO with 2 step oxidation (Step 1 material oxidized again using the same procedure). In particular, cooling experiments in 1-alcohols were performed at ESRF using Step $1 \mathrm{~B}-\mathrm{GO}$ with $\mathrm{C}: \mathrm{O}=2.9$, vacuum evaporation experiments using B-GO step 1 with $\mathrm{C} / \mathrm{O}=2.9$, TGA, DSC and isopiestic experiments were performed using Step 1 and Step 2 B-GO samples with C:O equal to 2.8 and 2.6, respectively. Averaged C:O for five batches was $2.7 \pm 0,2$.

Figures S1 and S2 present XPS spectra of B-GO 1step and 2 step samples used in this study. The spectra were recorded with a Kratos Axis Ultra electron spectrometer equipped with a delay line detector. A monochromated Al KR source operated at 150W, a hybrid lens system with a magnetic lens, providing an analysis area of $0.3 \mathrm{~mm} * 0.7 \mathrm{~mm}$, and a charge neutralizer were used for the measurements. The binding energy (BE) scale was adjusted with respect to the $\mathrm{C} 1 \mathrm{~s}$ line of aliphatic carbon, set at $285.0 \mathrm{eV}$.

The assignments of $\mathrm{C} 1 \mathrm{~s}$ peaks were taken from ref. ${ }^{1}$. 


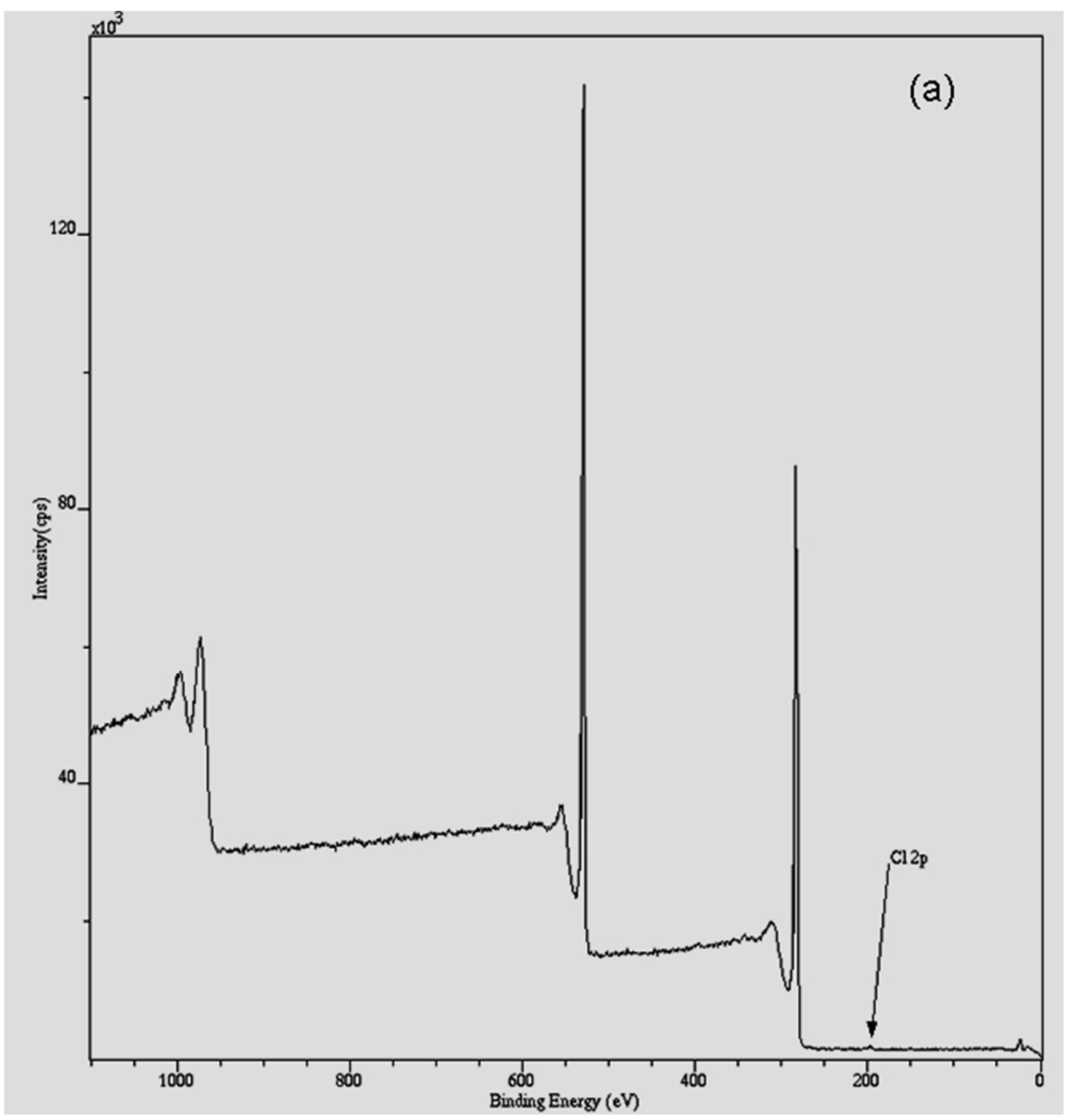




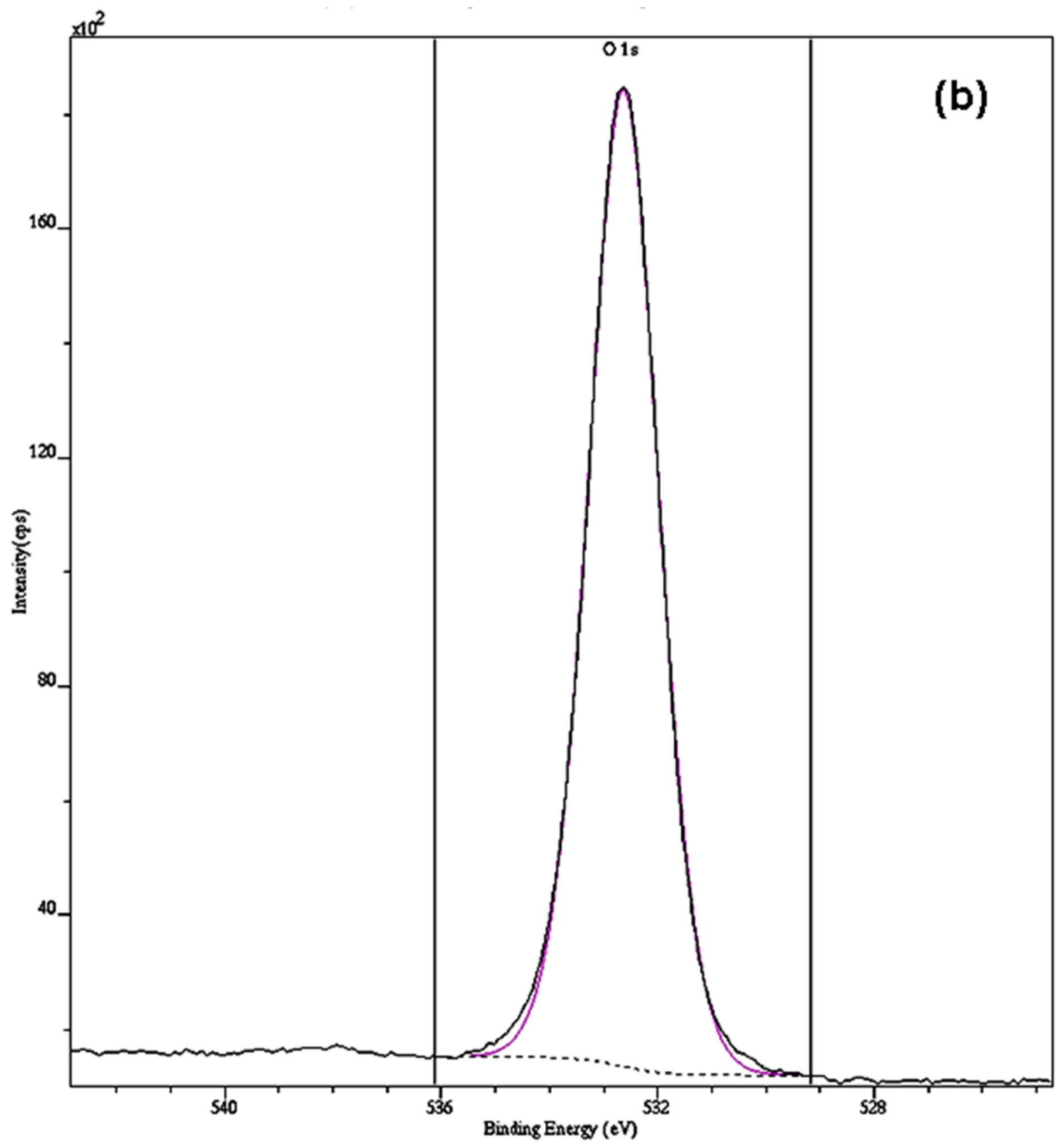

S 3 


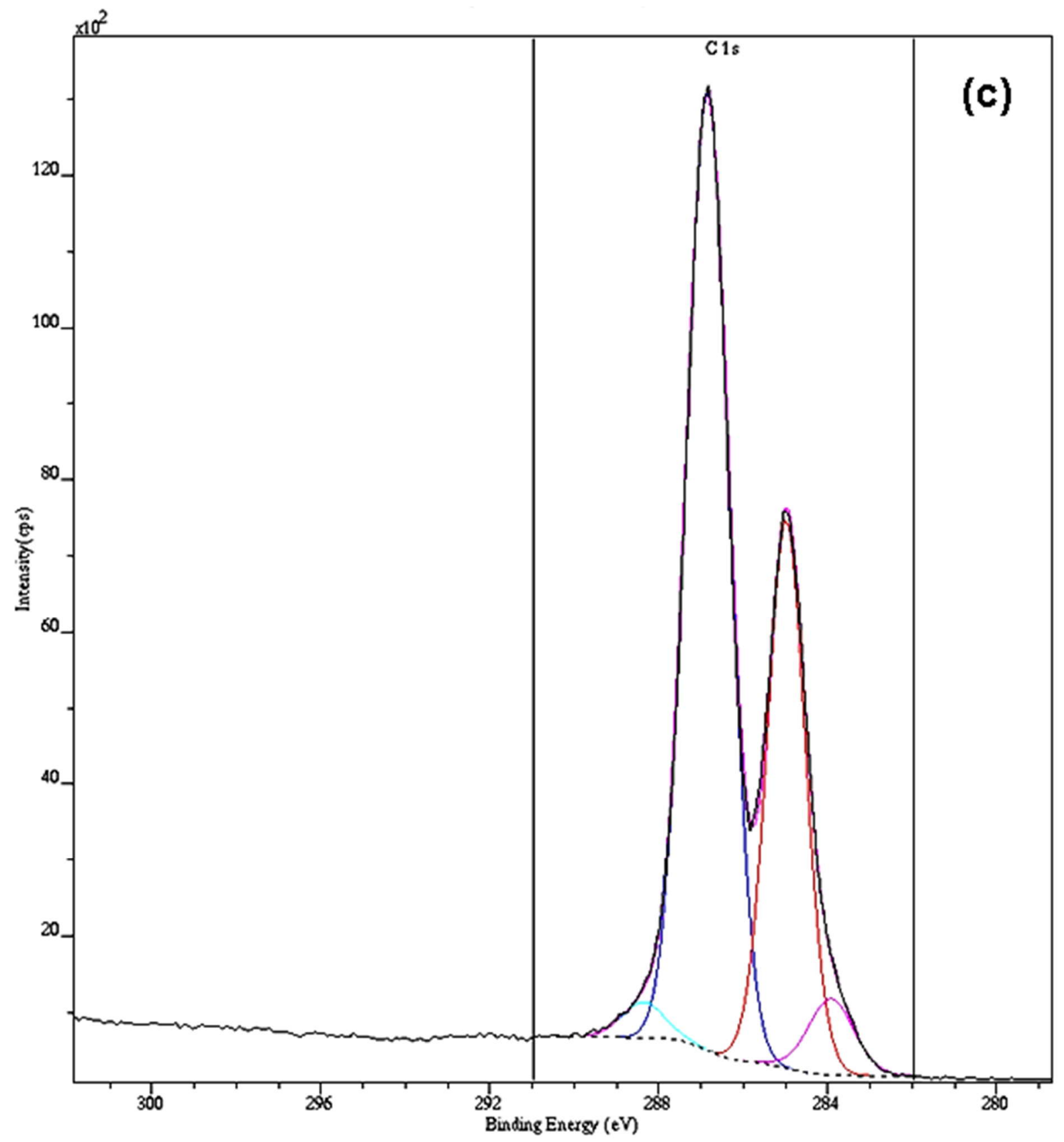

Fig. S1. XPS spectrum of the B-GO sample produced by one-step oxidation.

a) XPS spectrum; b) O1s peak c) C 1s peak. 
Table S1. XPS spectra of the sample produced by one-step oxidation.

\begin{tabular}{|c|c|c|c|c|c|}
\hline Line & $\begin{array}{l}\text { Binding } \\
\text { energy, eV }\end{array}$ & $\begin{array}{l}\text { Possible } \\
\text { assingment }\end{array}$ & FWHM, eV & at $\%$ & $\mathrm{C}: \mathrm{O}$ \\
\hline \multirow[t]{5}{*}{$\mathrm{C} 1 \mathrm{~s}$} & 283.9 & Unclear (?) & 1.25 & 3.82 & \multirow{6}{*}{2.80} \\
\hline & 285 & $\mathrm{C}-\mathrm{C}$ & 1.05 & 22.96 & \\
\hline & 286.9 & $\mathrm{C}-\mathrm{O}-\mathrm{C} ; \mathrm{C}-\mathrm{OH}$ & 1.15 & 45.25 & \\
\hline & 288.4 & $\mathrm{HO}-\mathrm{C}=\mathrm{O}$ & 1.15 & 1.65 & \\
\hline & & & & Total:73.68 & \\
\hline O 1s & 532.6 & & 1.5 & 26.32 & \\
\hline
\end{tabular}

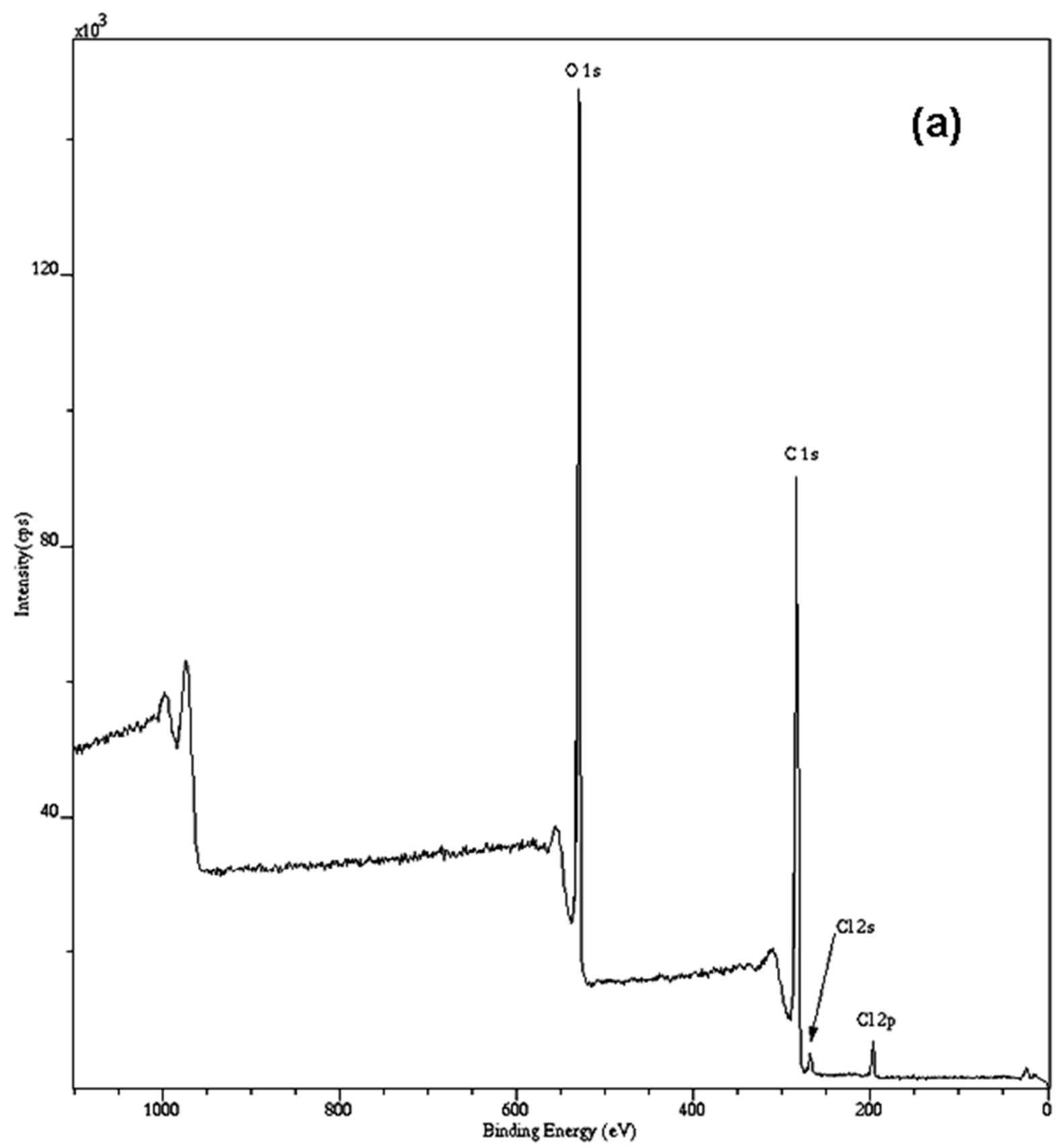




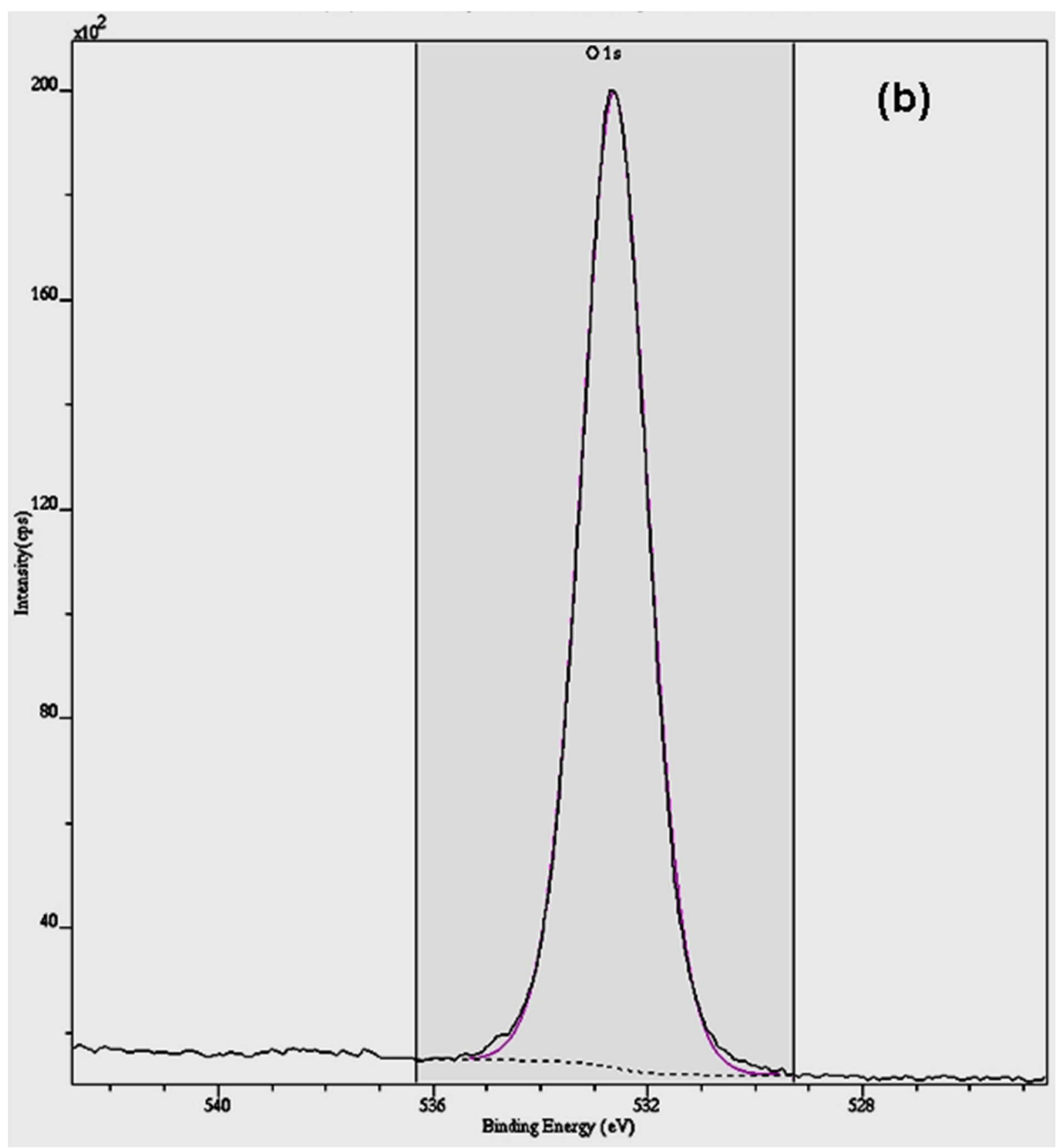




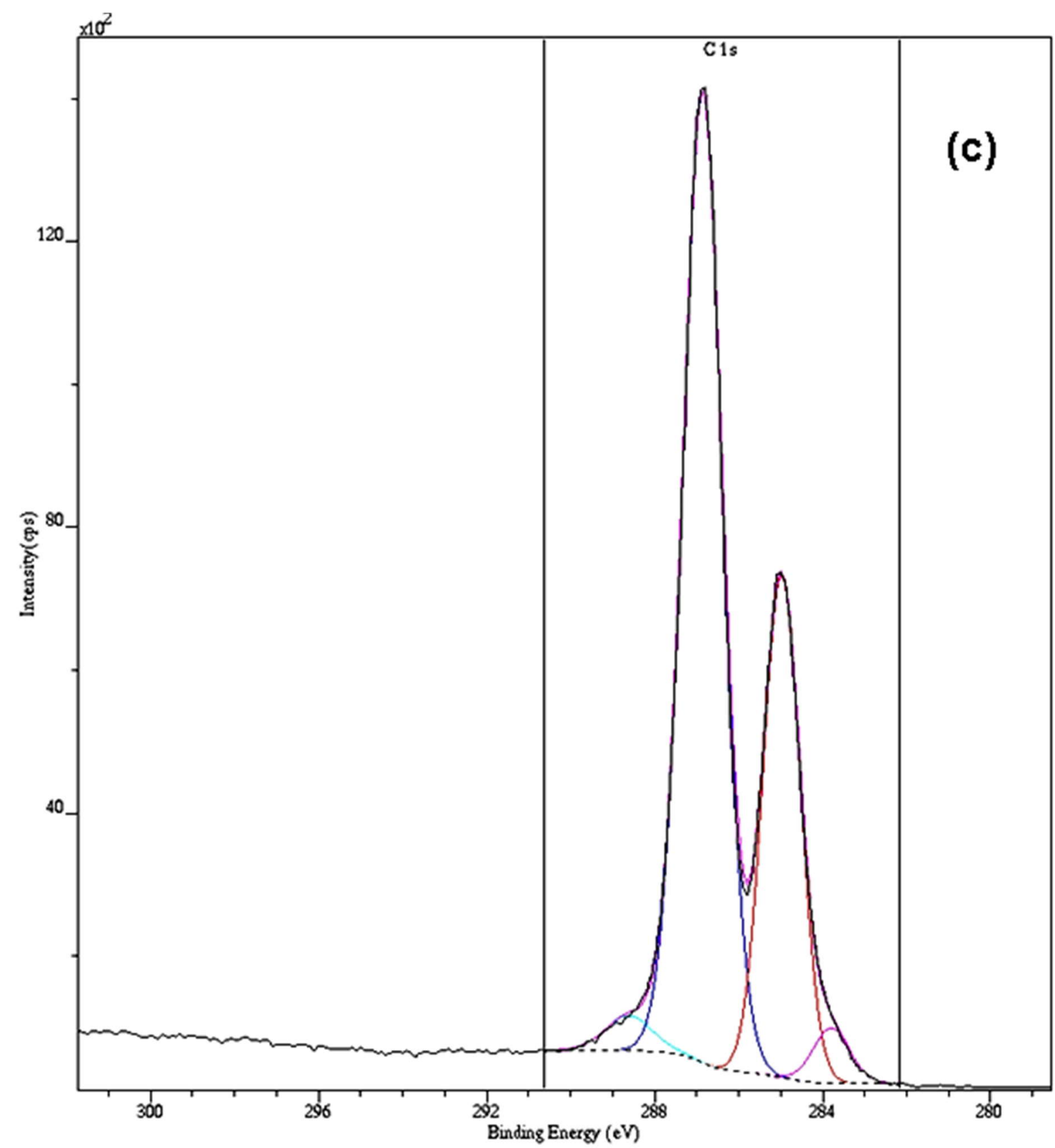

Fig. S2. XPS spectra of the sample produced by two step oxidation.

a) XPS spectrum; b) O1s peak c) C 1s peak. 
Table S2. XPS spectra of the sample produced by two step oxidation.

\begin{tabular}{|c|l|l|c|c|c|}
\hline Line & $\begin{array}{l}\text { Binding } \\
\text { energy, eV }\end{array}$ & $\begin{array}{l}\text { Functional } \\
\text { groups }\end{array}$ & FWHM, eV & at\% & C:O \\
\hline \multirow{2}{*}{ C 1s } & 283.8 & Unclear (?) & 1 & 2.34 & \\
\cline { 2 - 5 } & 285 & C-C & 1 & 21.5 & \multirow{2}{*}{2.59} \\
\cline { 2 - 6 } & 287 & C-O-C; C-OH & 1.1 & 45.47 & \\
\cline { 2 - 6 } & 289 & HO-C=O & 1,3 & 1.91 & \\
\cline { 2 - 6 } & & & 1.45 & 27.53 & \\
\hline O 1s & 532.6 & & & & \\
\hline
\end{tabular}

\section{S2. Results and Discussion.}

2S.1. Isothermal Thermogravimetry (TG)

Isothermal Termograviametric (TG) runs were carried out with the samples of the systems B-GO - 1-butanol, 1- pentanol, 1-hexanol (see fig.2S, a,b,c) and 1-heptanol (see fig.1 of the main text). Desorption of alcohols occurred from the cylindrical cells with the diameter $\mathrm{D}$, through the small orifice with the diameter $\mathrm{d}$. The ratio of $\mathrm{D} / \mathrm{d}$ was about 50 . When the same desorption occurred from the open cells under non-equilibrium conditions the step-wise desorption was not observed and the decrease of mass was monotonous.

Three portions of the traces parallel to t-axes in fig.S2 a,b,c correspond to the desorption of alcohols from different heterogeneous two-phase regions of the binary phase diagrams. When desorbing alcohol the system goes from the two phase region $\{$ pure normal alcohol $+(\mathrm{III})\}$ to phase region $\{(\mathrm{III})+(\mathrm{II})\}$ and then to $\{(\mathrm{II})-(\mathrm{I})\}$. Roman numbers denote different swollen structures. Vertical portions of the TG curves correspond to crossing the compositions of the swollen structures (III) and (II) (see the main text). 

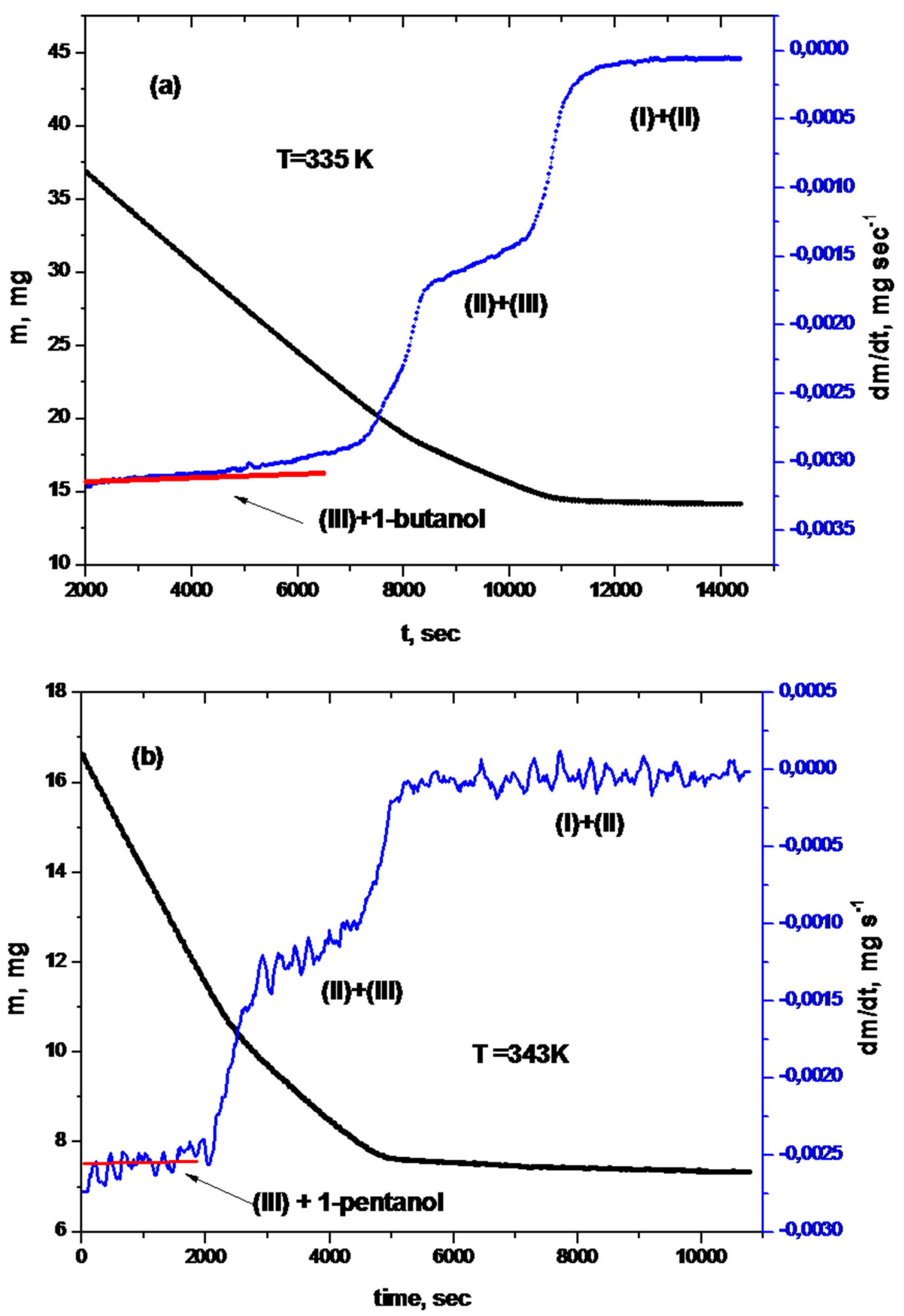


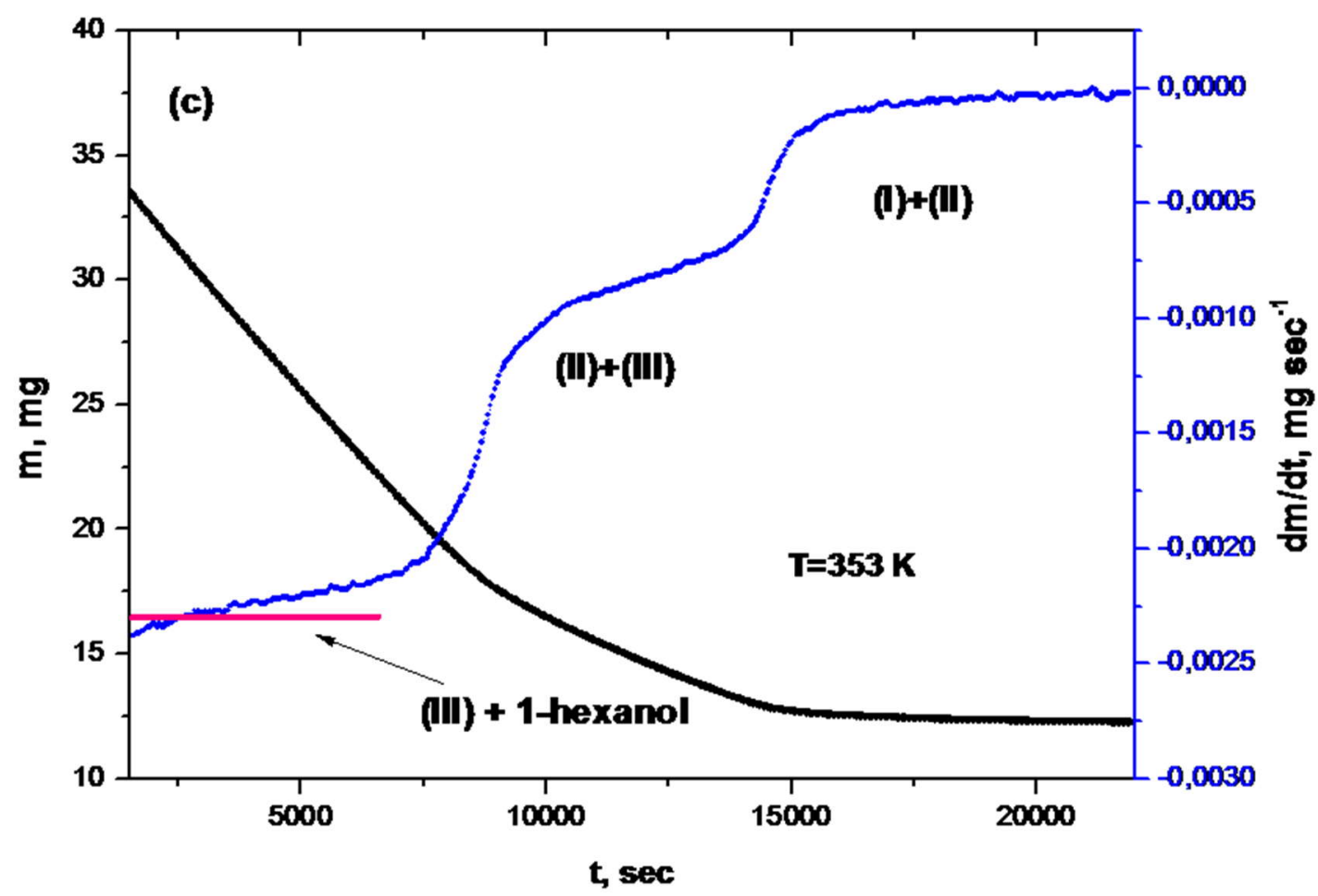

Fig.S3. Isothermal desorption in the systems a) B-GO - 1-butanol, T=335 K; b) B-GO 1pentanol, $\mathrm{T}=343 \mathrm{~K}$; c) B-GO - 1- hexanol, T=353 K. Black line - mass of the system vs time, blue line - rate of desorption vs time, red line - rate of desorption of pure normal alcohol, taken from separate run performed under the same conditions.

2S.2.2 Additional XRD data for experiments with non-equilibrium vacuum drying of solvents from the systems $B-G O$ - normal alcohol and for B-GO cooling/heating in various normal alcohols in the closed cell under equilibrium conditions. 


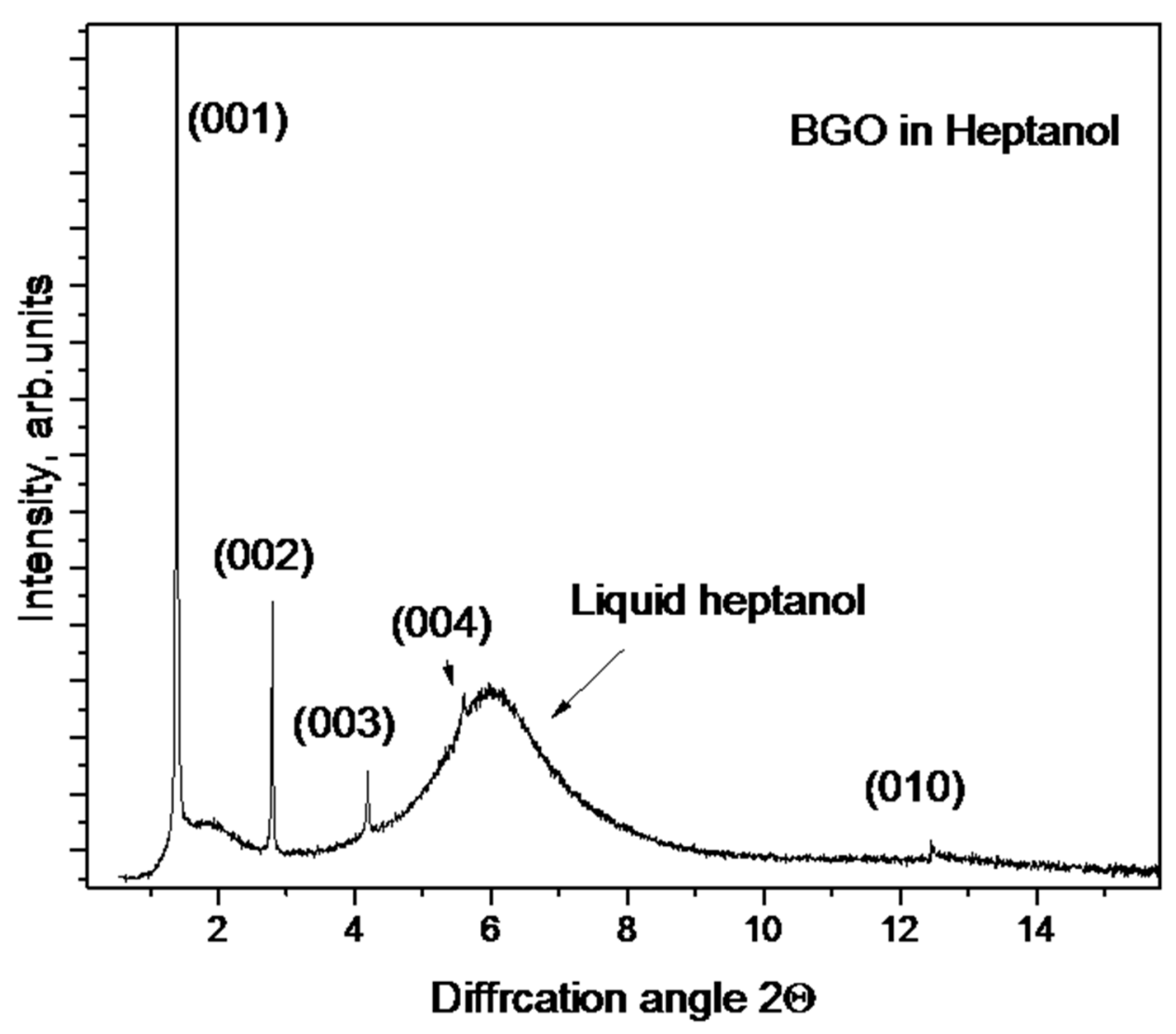

Figure 4S. Example of XRD pattern recorded in transmission mode inside of glass capillary filled

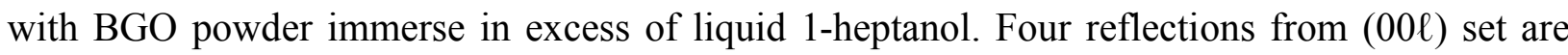
observed. Broad peak is due to liquid heptanol, $\mathrm{T}=298 \mathrm{~K}$. Recorded using synchrotron radiation with $\lambda=0.46794 \AA$. 


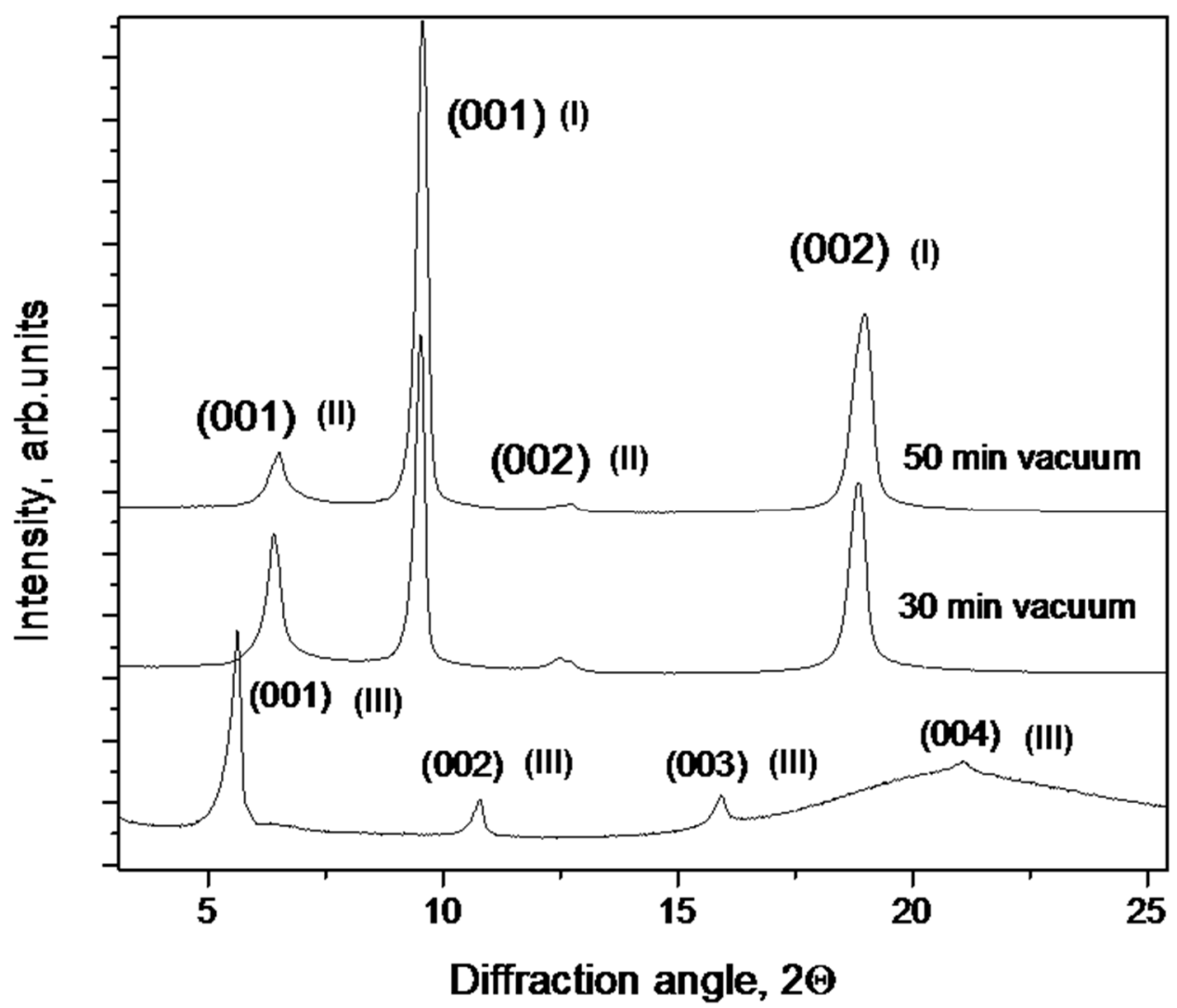

Figure S5. XRD patterns recoded from BGO in liquid 1-butanol in process of vacuum driven solvent evaporation $(\mathrm{CuK} \alpha)$

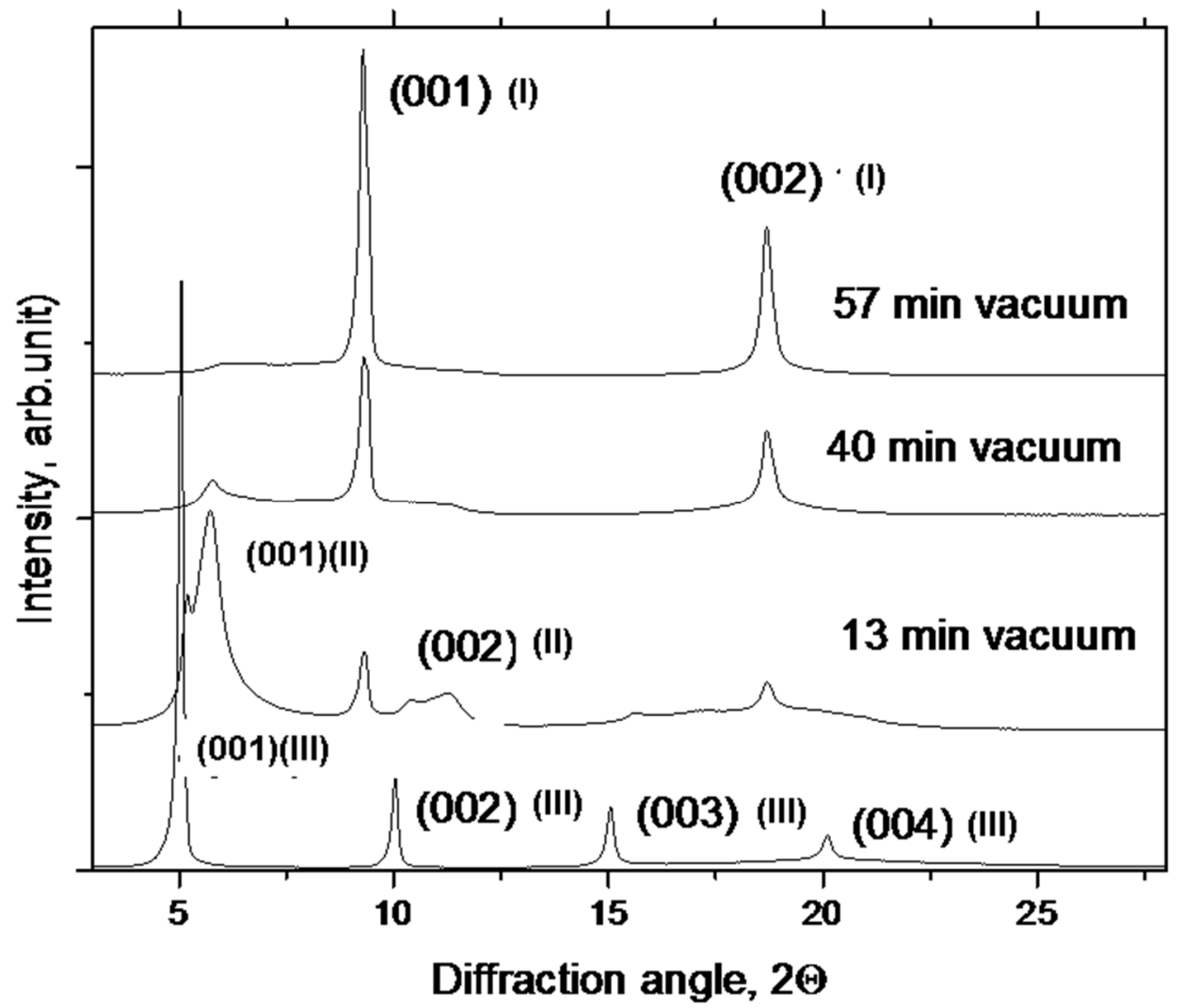


Figure S6 XRD patterns recoded from BGO in liquid 1-pentanol in process of vacuum driven solvent evaporation $(\mathrm{CuK} \alpha) . \mathrm{d}(001)(\mathrm{II})=15.42 \AA, \mathrm{d}(001)(\mathrm{III})=17.5 \AA$.

(a)

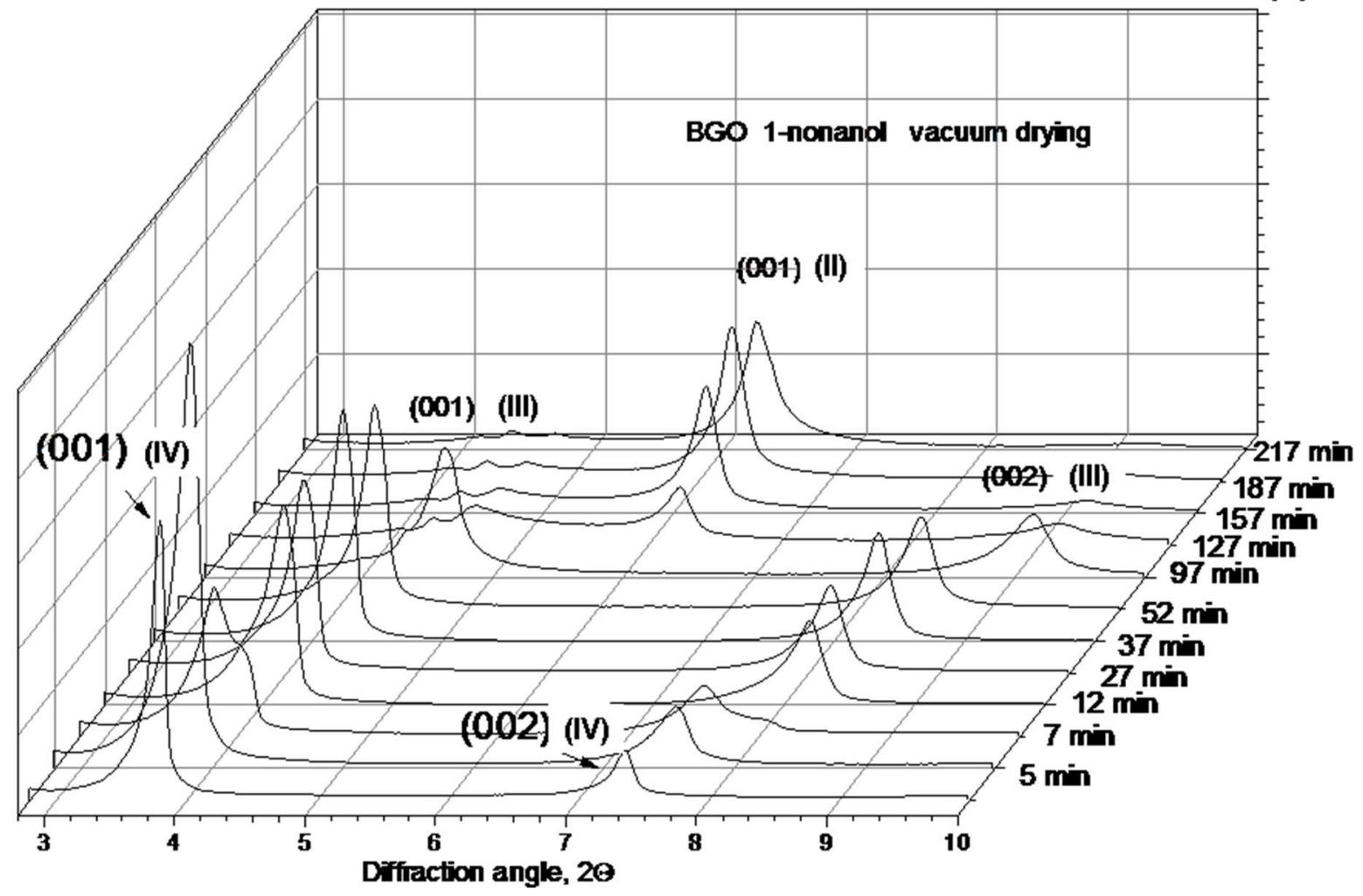

(b)

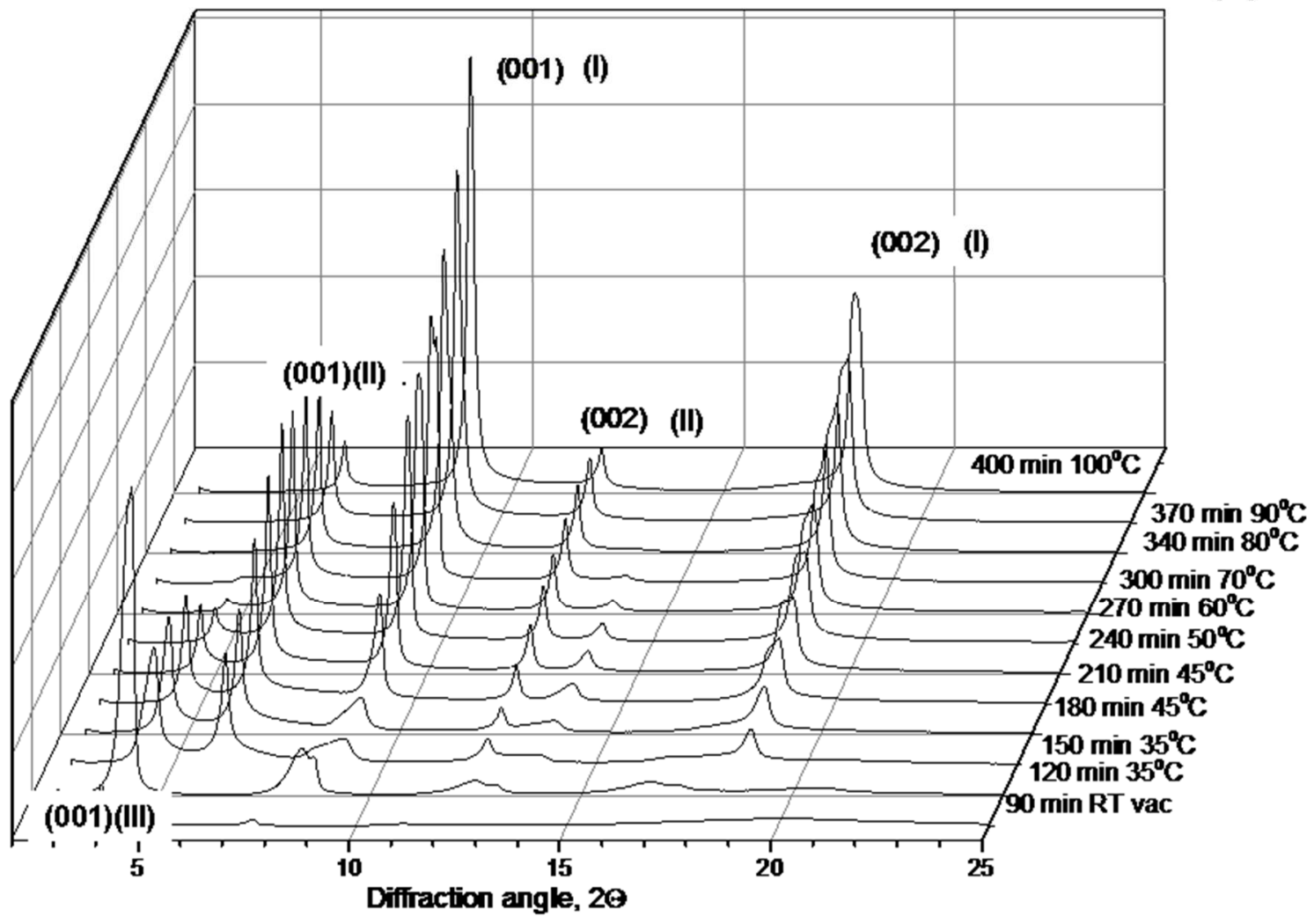


Figure S7 a,b. XRD patterns (not the whole range) recorded from BGO-1-nonanol in process of non-equilibrium vacuum drying $(\mathrm{CuK} \alpha)$.

a) The sample has excess of nonanol at the starting point and corresponds to swollen structure (IV). Vacuum driven evaporation at ambient temperature results in gradual transition into swollen structure (III) which is complete in the $97 \mathrm{~min}$ pattern. Further evaporation of solvent leads to formation of phase (II). Since the evaporation is rather slow at ambient temperature, the experiment was stopped after $217 \mathrm{~min}$ and repeated with adding heating (see Figure S3b)

b) XRD patterns recorded in process of vacuum drying of BGO-1-nonanol at elevated temperatures $(\mathrm{CuK} \alpha)$. The solvent free structure was not recovered even after vacuum heating at $100^{\circ} \mathrm{C}$. Degradation of GO starts above $\sim 110^{\circ} \mathrm{C}$, therefore the heating was stopped below this temperature.

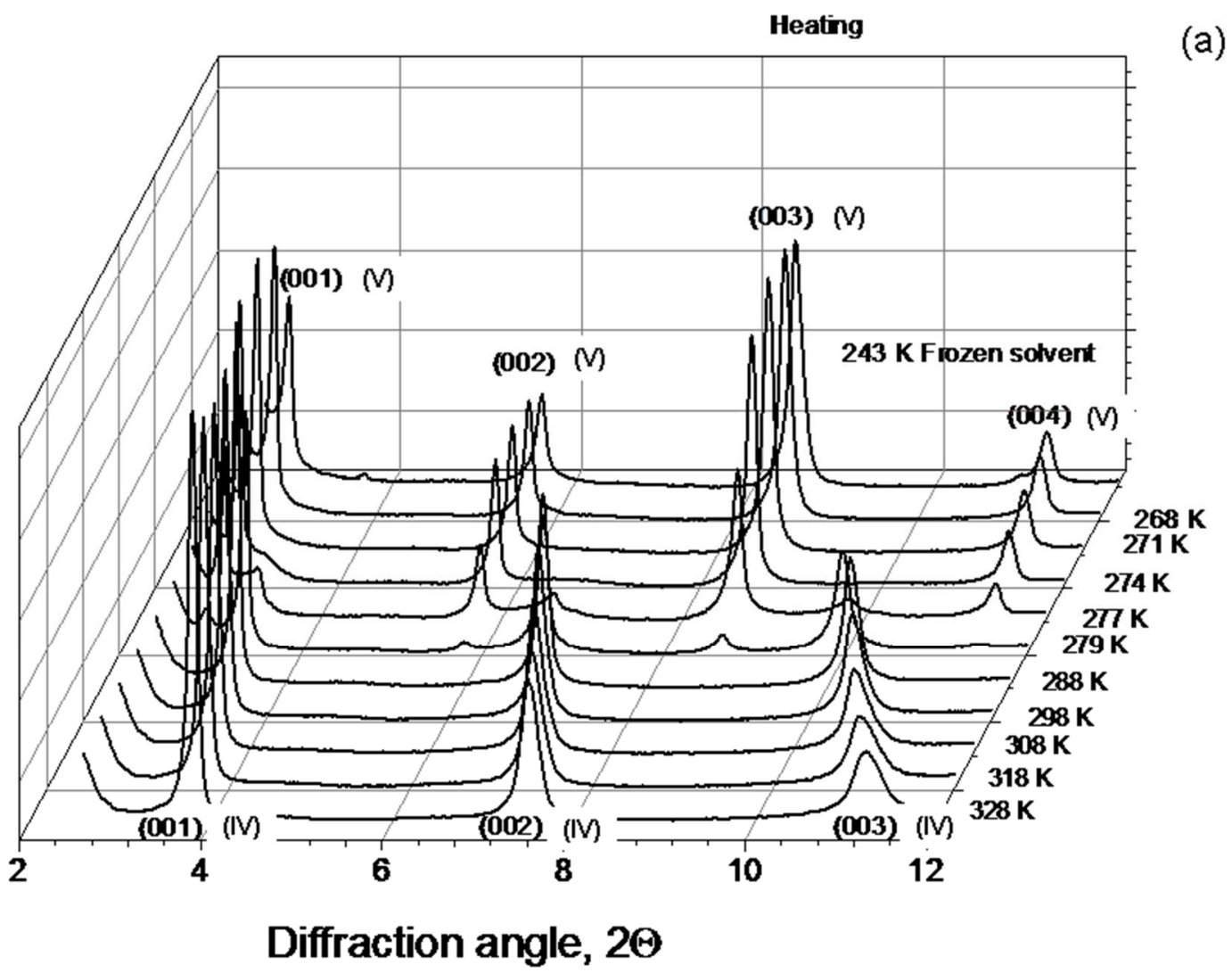




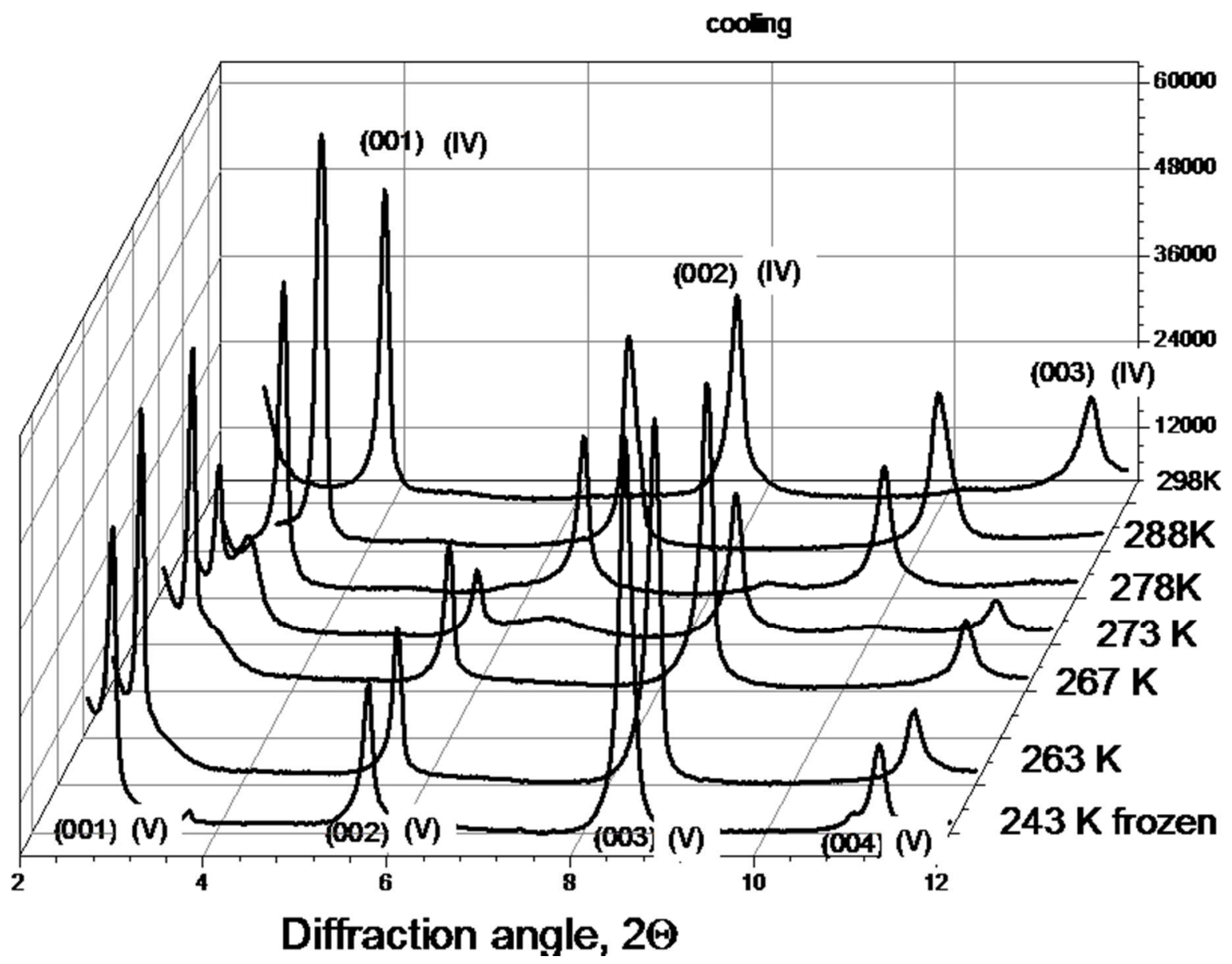

(b)

Figure S8. a,b. XRD patterns recorded from BGO in liquid 1-nonanol $(\mathrm{CuK} \alpha)$ in heating (a) and colling (b) around a temperature of incongruent melting phase transition $(\mathrm{T}=284 \mathrm{~K}$ according to DSC. See the main text).

S2.3. Determination of sorption and compositions of the swollen structures from the DSC data.

Fig. S9 a,b present the DSC trace in the system $\mathrm{BGO}$ - 1- nonanol. The sample consisted of the B-GO powder and the excess of the pure 1-nonanol. The initial amounts of both components $\mathrm{m}_{B-G O}$ and $\mathrm{m}_{1-n o n a n o l}$ were determined by weighing. The peak at $\mathrm{T}=268 \mathrm{~K}$ corresponded to the melting of free 1-nonanol that was not sorbed into B-GO. The second peak at $\mathrm{T}=284 \mathrm{~K}$ was attributed to incongruent melting of the swollen structure (V) (see the Main text). The initial amount of 1-nonanol in the sample was distributed between two phases, namely, the swollen structure (V) and the pure liquid 1-nonanol. Sorption of 1-nonanol into SwSt (V), $\mathrm{m}_{\text {l-Nonanol }}$ (V), was calculated from the equation 


$$
m_{1-\text { nonanol }}(V)=m_{1-\text { nonanol }}-\frac{Q_{1}}{\Delta H_{s p}}
$$

where $\mathrm{Q}_{1}, \Delta \mathrm{H}_{s p}$, are the enthalpy of melting calculated from the integrated area of the DSC peak at $268 \mathrm{~K}$ and the enthalpy of melting of pure 1-nonanol, measured in this study as $24 \pm 1 \mathrm{~kJ}$ $\mathrm{mol}^{-1}$. The enthalpy of melting of 1-nonanol was also estimated in ref. ${ }^{\mathrm{S} 2}$ as $23.2 \mathrm{~kJ} \mathrm{~mol}^{-1}$. , respectively. The sorption $\mathrm{m}_{\text {1-Nonanol }}(\mathrm{V})$ corresponded to the temperature of melting of 1nonanol. The whole amount of B-GO in the sample was incorporated into SwSt (V). The

composition of the swollen structure (V) was calculated from the mass ratio

$$
\frac{m_{1-\text { nonanol }}(V)}{m_{B-G O}}
$$

Measurements were performed with the samples of different initial composition. The averaged mass ratio was equal to 1.69 , which corresponds to molar ratio equal to 0.21 . The molar mass of $\mathrm{B}-\mathrm{GO}$ was taken to be $18 \mathrm{~g} \mathrm{~mol}^{-1}$ (see the main text). Briefly speaking, the initial composition of the sample at which peak at $268 \mathrm{~K}$ disappears from the DSC trace is equal to the composition of SwSt (V).

From the integrated area of the DSC peak, $\mathrm{Q}_{2}$ at $284 \mathrm{~K}$ the enthalpy of incongruent melting, $\Delta H_{\text {in }}$,was calculated as $17.6 \mathrm{~kJ} \mathrm{~mol}^{-1}$ of 1-nonanol.

The DSC traces presented in fig. S were observed for the samples with the initial mass ratio $\underline{m_{1-\text { nonanol }}}$

$m_{B-G O}>1.69$. If the initial mass ratio was smaller, peak at $\mathrm{T}=268 \mathrm{~K}$ disappeared from the DSC traces, because now there was no "non-sorbed" 1-nonanl in the system. The enthalpy $\mathrm{Q}_{2}$ became smaller than $\Delta H_{\text {in }}$. The initial amounts of both components, $\mathrm{m}_{B-G O}$ and $\mathrm{m}_{\text {1-nonanol }}$ were distributed now between phases (V) and (IV). The following set of mass balance equations was valid

$$
\begin{aligned}
& m_{B-G O}=m_{B-G O}(I V)+m_{B-G O}(V) ; \\
& m_{1-\text { nonanol }}=m_{1-\text { nonanol }}(I V)+m_{1-\text { nonanol }}(V) \\
& m_{1-\text { nonanol }}(V)=Q_{2} / \Delta H_{\text {in }} ; m_{1-\text { nonanol }}(V)=0.21 \times m_{B-G O}(V)
\end{aligned}
$$

Composition of SwSt (IV) was calculated from these equations as 1.16 (weight ratio of 1nonanol to B-GO) or as 0.14 (molar ratio of 1-nonanol to B-GO). Briefly speaking, the initial 
composition of the sample at which peak at $284 \mathrm{~K}$ disappears from the DSC trace is equal to the composition of SwSt (V)

The incongruent melting decomposition corresponds to the reaction (see eq. 1 in the main text) $\mathrm{BGO}\left(\mathrm{CH}_{3}\left(\mathrm{CH}_{2}\right)_{8} \mathrm{OH}\right)_{0.21} \rightarrow \mathrm{BGO}\left(\mathrm{CH}_{3}\left(\mathrm{CH}_{2}\right)_{8} \mathrm{OH}\right)_{0.14}+0.07\left(\mathrm{CH}_{3}\left(\mathrm{CH}_{2}\right)_{8} \mathrm{OH}\right.$

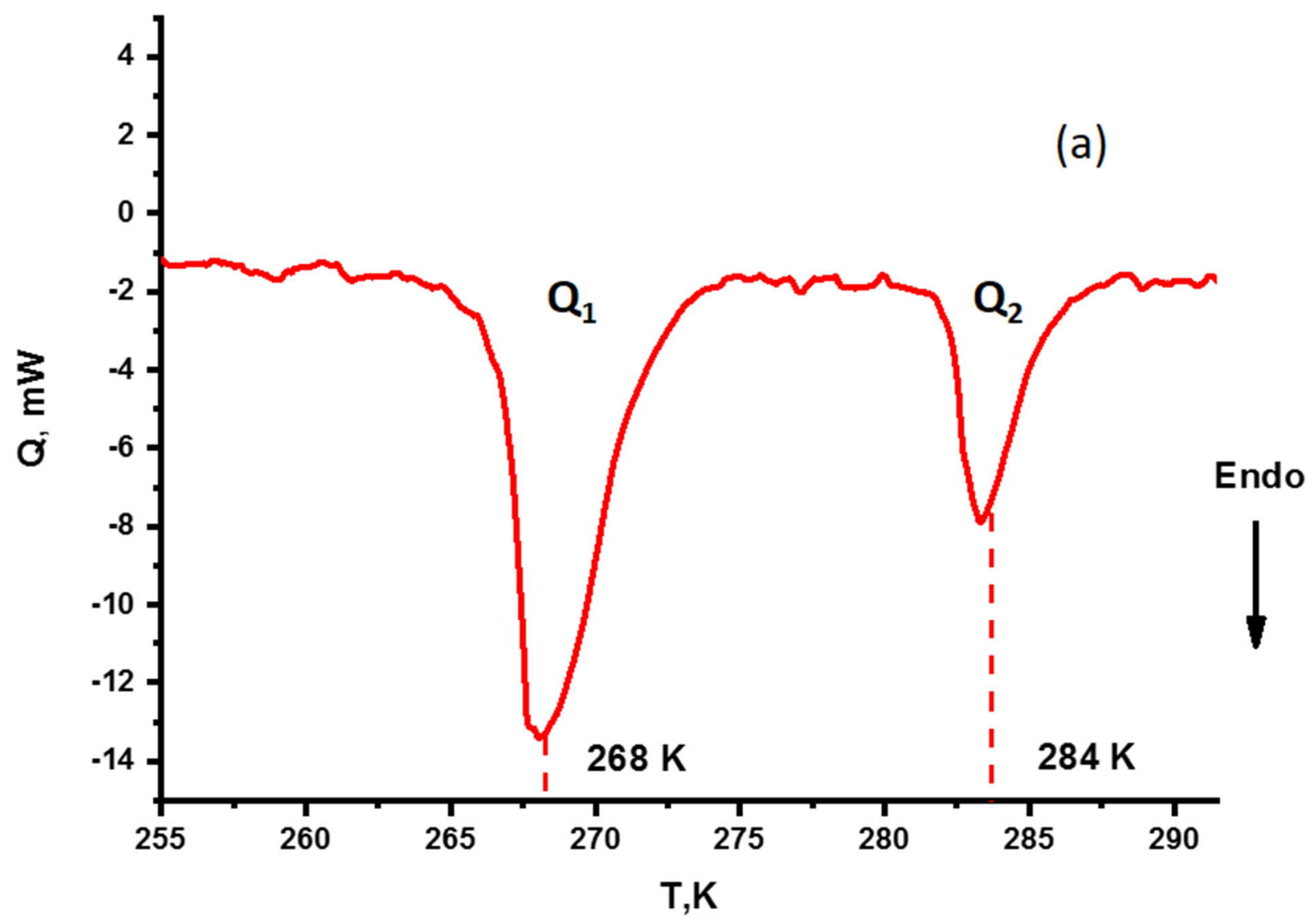




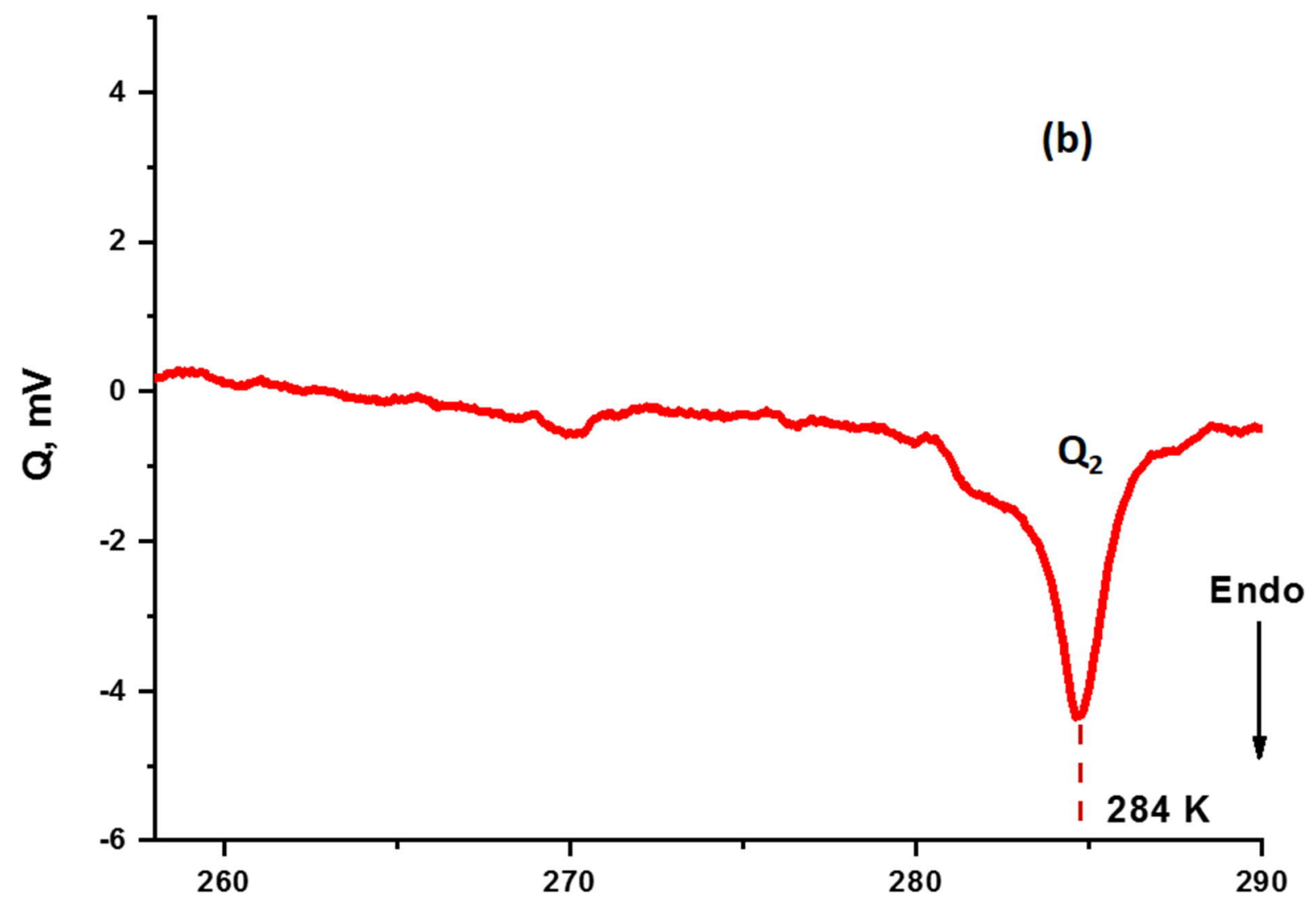

Fig.9S a,b. The system B-GO - 1-nonanol. (a) DSC trace for the sample with the mass ratio (1nonanol: $\mathrm{B}-\mathrm{GO}=3.1$ ). Peaks at 268, $284 \mathrm{~K}$ are melting of free 1-nonanol and incongruent melting of the SwSt (V), respectively. (b) DSC trace for the sample with the mass ratio (1nonanol: $\mathrm{B}-\mathrm{GO}=1.34)$. Peak at $284 \mathrm{~K}$ is incongruent melting of the SwSt (V).

References.

S1. You, S.; Luzan, S.; T. Szabo, T.; Talyzin, A.V. Effect of Synthesis Method on Solvation and Exfoliation of Graphite Oxide. Carbon 2013, 52, 171-180.

S2. https://www.chemeo.com/cid/39-947-8/1-Nonanol 